\title{
A Micro-manipulation Platform Based on Magnetic Tweezers and Total Internal Reflection Fluorescence Microscopy for Cell Mechanical Studies
}

\author{
Chaug-Chun Lee, Cheng-Hao Chien, Liang-Kung Wen, Chen-Yuan Dong* \\ Microscopic Biophysics Laboratory, Department of Physics, National Taiwan University, Taipei, Taiwan 106, \\ ROC
}

Tel: 886-2-33665196, Fax: 886-2-33665244, * E-mail: cydong@phys.ntu.edu.tw

\begin{abstract}
We developed a manipulation platform, which combines magnetic tweezers and the incubating system, with total internal reflection fluorescence microscopy (TIRFM). Our system can generate forces up to the $n N$ range and such forces can be used to observe and characterize cell mechanical responses to external forces. Combined with TIRFM, cell surface stress-strain phenomena can also be investigated.
\end{abstract}

\section{SUMMARY}

Purpose -- The mechanism of cell motility and cell growth is not completely understood. The investigation of cell motility and cell growth can improve the fundamental knowledge in cell biology, improve the tissue engineering protocols, and can be applied for developing an effective method to culture and to differentiate cells. To better understand cell mechanics, we apply well controlled, external mechanical forces on the cells in an effort to observe the influences of applied stress on the cells. From these results, we hope to gain more insight into the mechanism of cell motility and cell growth.

Instrument - This work is intended to describe the developments of the micro-manipulation system we have established. The instrument we have developed is shown in Fig. 1. By using a magnetic tweezers combined with total internal reflection fluorescence microscopy (TIRFM), we produced a system capable of exerting forces on cellular systems. By controlling the level of applied current to the electromagnetic, forces can be generated up to the $\mathrm{nN}$ range (see calibration data in Fig. 2). Shown in Fig. 3 are images of the magnetic beads attached to the cells and in Fig. 4 are TIRFM images of fluorescent microspheres.

We have also developed a microscope incubating system that can support long-term observation of living cells. To investigate cell surface events, TIRFM, known as a useful tool for the observation on the surface (within the evanescent field about $100 \mathrm{~nm}$ depth), is combined with the magnetic tweezers system to observe events in cell mechanics. Using this system, we plan to perform experiments to characterize the responses of the cell membranes under varying force/durations, and to understand the role of the external forces in cell motility and cell growth.

\section{Reference}




\section{THP-36(T7)}

[1] Fass and Odde, "Tensile Force-Dependent Neurite Elicitation via Anti- $\beta 1$ Integrin Antibody-Coated Magnetic Beads", Biophysical Journal, Vol. 85, 623-636, 2003

[2] Galbraith, Yamada, Sheetz, "The relationship between force and focal complex development", Journal of Cell Biology, Vol.159, 695-705, 2002

[3] Huang H, Dong CY, Kwon HS, Sutin JD, Kamm RD, So PTC, "Three-dimensional cellular deformation analysis with a two-photon magnetic manipulator workstation", Biophysical Journal, 82 (4): 2211-2223 APR 2002

[4] Huang H, Kamm RD, So PTC, Lee RT, "Receptor-based differences in human aortic smooth muscle cell membrane stiffness", Hypertension, 38 (5): 1158-1161 NOV 2001

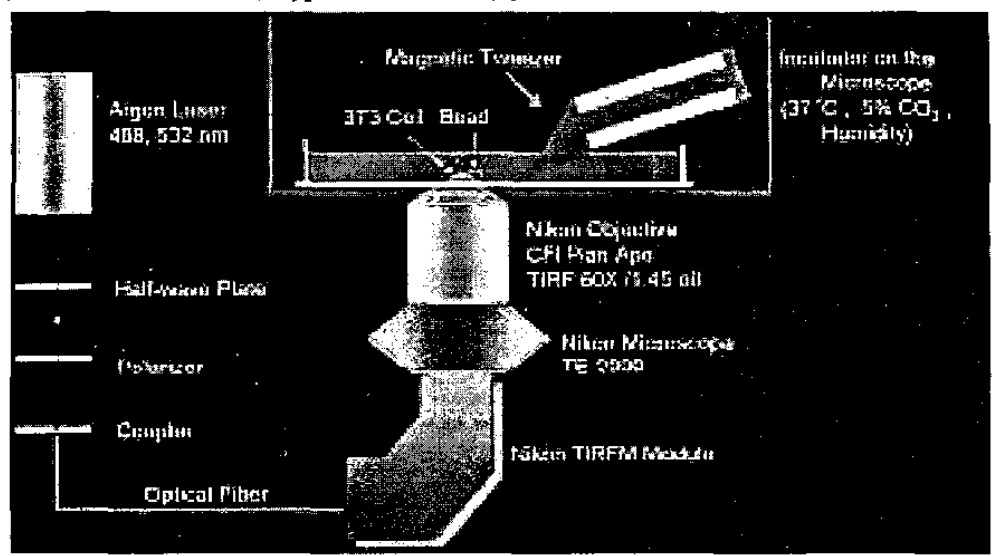

Fig1. The magnetic micro-manipulation, TIRFM microscope

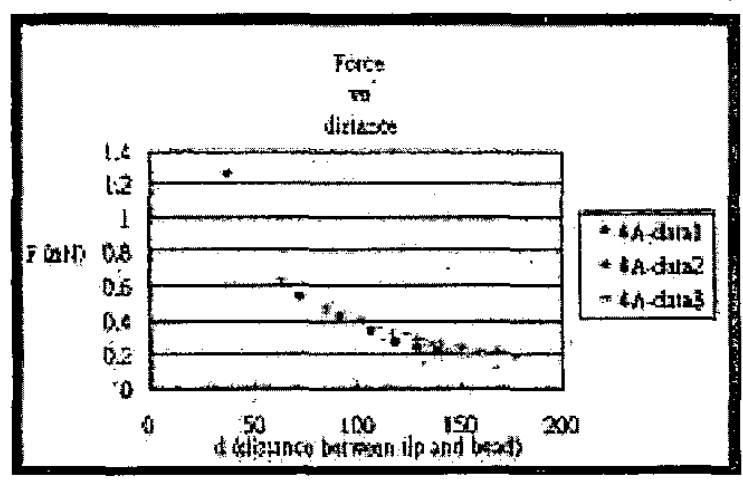

Fig2. Force calibration data

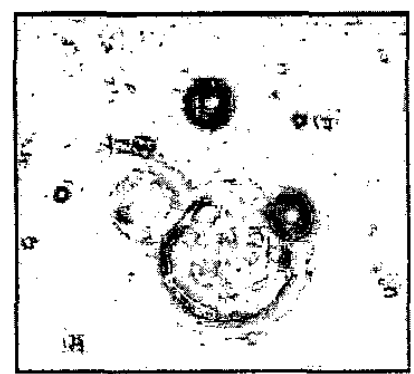

Fig3. Magnetic beads attached on PC-12 cells

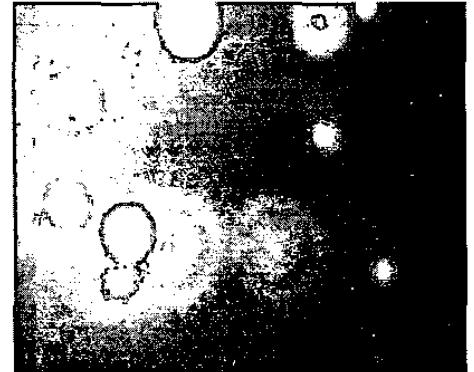

Fig4. TIRFM images of the microspheres (orange, $0.1 \mu \mathrm{m}$ ) 